\title{
Macroscopic aspects of the Unruh effect
}

\author{
Detlev Buchholz ${ }^{(a)}$ and Rainer Verch ${ }^{(b)}$ \\ (a) Institut für Theoretische Physik, Universität Göttingen, \\ Friedrich-Hund-Platz 1, 37077 Göttingen, Germany \\ (b) Institut für Theoretische Physik, Universität Leipzig, \\ Brüderstr. 16, 04103 Leipzig, Germany
}

\begin{abstract}
Macroscopic concepts pertaining to the Unruh effect are elaborated and used to clarify its physical manifestations. Based on a description of the motion of accelerated, spatially extended laboratories in Minkowski space in terms of Poincaré transformations, it is shown that, from a macroscopic perspective, an accelerated observer will not register with his measuring instruments any global thermal effects of acceleration in the inertial (Minkowskian) vacuum state. As is explained, this result is not in conflict with the well-known fact that microscopic probes used as thermometers respond non-trivially to acceleration if coupled to the vacuum. But this response cannot be interpreted as the effect of some exchange of thermal energy with a gas surrounding the observer; in fact, it is induced by the measuring process itself. It is also shown that genuine equilibrium states in a uniformly accelerated laboratory cannot be spatially homogeneous. In particular, these states coincide with the homogeneous inertial vacuum at sufficiently large distances from the horizon of the observer and consequently have the same (zero) temperature there. The analysis is carried out in the theory of a free massless scalar field; however the conclusion that the Unruh effect is not of a macroscopic thermal origin is generally valid.
\end{abstract}

\section{Introduction}

In spite of the fact that the computational aspects of the Unruh effect have been extensively studied and are by now well understood [28, 26, 32, there has not yet emerged a consensus on its proper physical interpretation, cf. for example [13, 9, 4]. It seems desirable to settle this matter in order 
to gain clarity as to what the theory actually predicts about the as yet unattainable experimental situation [6]. In the present article we elaborate theoretical concepts whose operational significance in the context of the Unruh effect is evident but whose mathematical description requires some care. Having clarified these concepts we will reconsider the theoretical predictions pertaining to the Unruh effect and come to conclusions that corroborate its dissenting interpretation brought forward in [4].

(i) The first point in the context of the Unruh effect which requires some thoughts is the fact that measuring devices are spatially extended. The popular idealisation that measurements are performed along world lines with comoving clocks attached to each line, indicating the respective proper time, corresponds neither to a realistic experimental situation nor to a meaningful theoretical hypothesis since it leads to difficulties when dealing with extended observables in the Heisenberg picture. Conceptual problems caused by this overidealized treatment were already observed by Bell, Hughes and Leinaas in [1]. We will therefore rely on the following more realistic scenario: A Minkowski space based observer enters with his clock a laboratory in a spacecraft that, at a given time, is at rest and then undergoes acceleration. The experimental equipment that he takes along is mounted to the walls of the laboratory at rest relative to him at all times, measured with his clock. Thus there is only one relevant time scale within the spatially extended laboratory. It is fixed by the clock of the observer and the interpretation of observations made with his measuring instruments rests on that time scale. It is understood that the walls of the laboratory are to be rigid in order to compensate tidal forces and allow for this standard experimental situation. We will discuss this point in the subsequent section, where we show that under mild constraints on the forces any motion of the laboratory can be described by a family of Poincaré transformations that is parametrised by the eigentime of the observer.

(ii) Next, there is the question at which scales observations are performed. Being interested in the Unruh effect, the observer will be led to analyse the macroscopic thermal properties of the vacuum in his laboratory. He does this by subsequent measurements of his observables in order to suppress microscopic fluctuations, thereby enhancing those features of the state that prevail at asymptotic times and hence can be interpreted as macroscopic (superselected) properties of the state [15, 22]. We will show in Sec. 3 that, irrespective of the motion performed by the laboratory, all observables form central sequences at asymptotic times in the state space of the Minkowski vacuum and have sharp (non-fluctuating) limits. The numerical values of these limits do not depend on the details of the motion and coincide with those found by an inertial observer. In other words, an accelerated observer will not register any macroscopic thermal effects caused by acceleration.

(iii) Having established the absence of thermal effects in the inertial vacuum which are caused by acceleration, the observer can prepare and study other states that are in equilibrium in his laboratory and analyse their macroscopic thermal properties. Restricting attention to the case of constant acceleration 
we will show in Sec. 4 that all genuine equilibrium states, characterised by the KMS-condition with respect to the accelerating dynamics, exhibit macroscopic properties that depend on the distance from the (apparent) horizon of the observer. Moreover, all equilibrium states coincide with the homogeneous inertial vacuum if restricted to compact regions of arbitrary size at sufficiently large distances from this horizon. Thus all equilibrium states have the same (zero) temperature in these remote regions. These facts, akin to the classical Tolman-Ehrenfest effect [27, 10], corroborate the assertion in [4] that the equilibrium parameters $T$ characterising KMS states cannot be interpreted as "local temperature" in the presence of acceleration; instead, they subsume information about the relation between the temperature and the acceleration that must prevail in order to accomplish global equilibrium.

(iv) Finally, the uniformly accelerated observer can place microscopic probes into equilibrium states, prepared in his laboratory, and determine at sufficiently large times the accumulated impact on the probes caused by the interaction with the states. Within the theoretical framework this situation is described by Pauli-Fierz type models of a finite dimensional quantum system that is weakly coupled to a macroscopic KMS state. In these models one can show under mild conditions on the underlying dynamics and couplings, applying to the case at hand, that the composed system approaches at large times a KMS state with the same equilibrium parameter $T$ as that of the initially unperturbed KMS state, cf. [8] and references quoted there. A detailed discussion of the Unruh effect in this setting has been given in [7]. From a physical point of view this "return to equilibrium" is not surprising. Yet this feature does not imply that the equilibrium parameter $T$ displayed by the microscopic probe can be interpreted as temperature of the macroscopic state, even if one corrects this reading by redshift factors depending on the position of the probe within the laboratory. For the interaction between the probe and the state does not only induce the desired exchange of thermal energy between the two systems; it also creates from this state additional excitations due to its local nature (cf. Reeh-Schlieder theorem [14, Sec. II.5.3]). These excitations transmit additional energy to the probe, proportional to the acceleration, leading to higher values of $T$ than the actual temperature of the macroscopic equilibrium state. So probes may not be regarded as perfect thermometers in the presence of acceleration since inevitable quantum effects in the measuring process affect their readings.

We conclude this introduction by defining our notation and presenting a model that is commonly used in discussions of the Unruh effect. Throughout this article we use units where $c=\hbar=k=1$. We consider four dimensional Minkowski space $\mathcal{M}=\left(\mathbb{R}^{4}, g\right)$ with proper coordinates $x=\left(x_{0}, \boldsymbol{x}\right)$ and metric $g$ fixed by the Lorentz scalar product $x \cdot y \doteq x_{0} y_{0}-\boldsymbol{x} \boldsymbol{y}$, where $\boldsymbol{x} \boldsymbol{y}$ denotes the Euclidean scalar product of the spatial components $\boldsymbol{x}, \boldsymbol{y}$. On $\mathcal{M}$ there acts the extended Poincaré group (Weyl group) $\mathscr{W}_{+}^{\uparrow} \doteq \mathbb{R}^{4} \rtimes\left(\mathscr{L}_{+}^{\uparrow} \times \mathbb{R}_{+}\right)$consisting of spacetime translations, proper orthochronous Lorentz transformations and dilations. The product of its elements $\Upsilon=(y, \Lambda, \lambda)$ is defined by 
$\Upsilon_{1} \Upsilon_{2}=\left(y_{1}, \Lambda_{1}, \lambda_{1}\right)\left(y_{2}, \Lambda_{2}, \lambda_{2}\right) \doteq\left(y_{1}+\Lambda_{1} \lambda_{1} y_{2}, \Lambda_{1} \Lambda_{2}, \lambda_{1} \lambda_{2}\right)$ in an obvious notation.

The model under consideration is the theory of a free massless scalar and hermitian field $\phi$. Since we consider several inequivalent representations of this field that are induced by different equilibrium states it is convenient to present the theory in terms of bounded functions $W(f)=\exp (i \phi(f))$ of the field, smeared with real test functions $f$ (Weyl operators). The resulting algebraic structures can be described as follows. Let $\mathcal{D}\left(\mathbb{R}^{4}\right)$ be the space of real valued test functions with compact support in $\mathbb{R}^{4}$ and let $\mathcal{D}_{\square}\left(\mathbb{R}^{4}\right) \doteq \mathcal{D}\left(\mathbb{R}^{4}\right) / \square \mathcal{D}\left(\mathbb{R}^{4}\right)$ be its quotient with regard to test functions lying in the kernel of the field (that fulfils the wave equation $\square \phi=0, \square$ being the d'Alembertian). We consider the *-algebra $\mathcal{A}\left(\mathbb{R}^{4}\right)$, which is generated by all sums and products of the operators $W(f), f \in \mathcal{D}_{\square}\left(\mathbb{R}^{4}\right)$, satisfying the Weyl relations

$$
W(f) W(g)=e^{-\kappa(f, g) / 2} W(f+g), \quad W(f)^{*}=W(-f),
$$

where $\kappa(f, g) \doteq(2 \pi)^{-3} \int d^{4} p \varepsilon\left(p_{0}\right) \delta\left(p^{2}\right) \widehat{f}(-p) \widehat{g}(p)$ is the commutator function of the field and $\widehat{f}, \widehat{g}$ are the Fourier transforms of (any member of the classes) $f, g \in \mathcal{D}_{\square}\left(\mathbb{R}^{4}\right)$. We also consider the subalgebras $\mathcal{A}(\mathcal{O}) \subset \mathcal{A}\left(\mathbb{R}^{4}\right)$, associated with double cones $\mathcal{O} \subset \mathbb{R}^{4}$, which are generated by the unitaries $W(f), f \in \mathcal{D}_{\square}(\mathcal{O})$, where $\mathcal{D}_{\square}(\mathcal{O}) \subset \mathcal{D}_{\square}\left(\mathbb{R}^{4}\right)$ denotes the class of test functions containing members, which have support in $\mathcal{O}$. It follows immediately from the properties of the commutator function that all elements of $\mathcal{A}\left(\mathcal{O}_{1}\right)$ commute with those of $\mathcal{A}\left(\mathcal{O}_{2}\right)$ if the double cones $\mathcal{O}_{1}, \mathcal{O}_{2}$ are spacelike separated (Einstein causality) or timelike separated (Huygens' principle). The extended Poincaré group acts on the algebra $\mathcal{A}\left(\mathbb{R}^{4}\right)$ by automorphisms. Since for any $\Upsilon=(y, \Lambda, \lambda) \in \mathscr{W}_{+}^{\uparrow}$ the linear map $f(x) \mapsto f_{\Upsilon}(x) \doteq \lambda^{-3} f\left(\Lambda^{-1} \lambda^{-1}(x-y)\right)$ on $\mathcal{D}\left(\mathbb{R}^{4}\right)$ leaves the subspace $\square \mathcal{D}\left(\mathbb{R}^{4}\right)$ and the commutator function invariant, the automorphic action is consistently defined by $\alpha_{\Upsilon}(W(f)) \doteq W\left(f_{\Upsilon}\right)$, $f \in \mathcal{D}_{\square}\left(\mathbb{R}^{4}\right)$. It follows from this definition that the group acts also covariantly, i.e. for any double cone $\mathcal{O} \subset \mathbb{R}^{4}$ one has $\alpha_{\Upsilon}(\mathcal{A}(\mathcal{O}))=\mathcal{A}(\Upsilon \mathcal{O})$, where $\Upsilon \mathcal{O} \doteq(\Lambda \lambda \mathcal{O}+y)$ in an obvious notation. Finally, the Minkowskian vacuum state $\omega_{0}$ on $\mathcal{A}\left(\mathbb{R}^{4}\right)$ is fixed by (linear extension from) the expectation values

$$
\omega_{0}(W(f)) \doteq e^{-v(f, f) / 2}, \quad f \in \mathcal{D}_{\square}\left(\mathbb{R}^{4}\right)
$$

where $v(f, f) \doteq(2 \pi)^{-3} \int d^{4} p \theta\left(p_{0}\right) \delta\left(p^{2}\right) \widehat{f}(-p) \widehat{f}(p)$ is the two-point function of the free field. The vacuum state is distinguished by the fact that it is invariant under the action of the extended Poincaré group, $\omega_{0} \circ \alpha_{\Upsilon}=\omega_{0}$ for $\Upsilon \in \mathscr{W}_{+}^{\uparrow}$, and that it is a ground state for the time translations in any Lorentz system. We will also consider thermal equilibrium (KMS) states on $\mathcal{A}\left(\mathbb{R}^{4}\right)$. By the Gelfand-NaimarkSegal (GNS) construction one can recover from any such state a concrete representation of the algebra on some Hilbert space. In case of the vacuum state $\omega_{0}$ one obtains the familiar Fock representation of the free field as well as a continuous unitary representation of $\mathscr{W}_{+}^{\uparrow}$, which induces in this representation 
the automorphic action of this group and satisfies the relativistic spectrum condition. We will make use of these facts in subsequent sections.

\section{Accelerated laboratories}

We turn now to the description of the motion of a laboratory which initially occupies the spherical region $\boldsymbol{L}_{0}=\left\{x: x_{0}=0,|\boldsymbol{x}-\boldsymbol{o}|<r\right\}$ of the time $x_{0}=0$ plane in the chosen Lorentz system. Its center $\boldsymbol{o}$ is the position of the observer carrying along a clock. As outlined in the introduction, we consider motions under the influence of arbitrary (not necessarily constant) accelerations. We do this by taking any sufficiently regular timelike and future directed world line of the observer as input. Disregarding spatial rotations for simplicity, the motion of the laboratory is described by the Fermi-Walker transport along that curve. There arise then the questions up to which magnitude of acceleration this idealised model of a laboratory is meaningful and whether its motion can be described by the action of Poincaré transformations.

The mathematical formulation of this issue proceeds as follows. Let $\gamma: t \mapsto \gamma(t)$ be the world line of the observer, parametrised by his proper time $t \geq 0$; it satisfies the initial conditions $\gamma(0)=(0, \boldsymbol{o})$, $\dot{\gamma}(0)=(1,0)$, where the dot indicates the derivative with respect to $t$. The curve is assumed to be timelike, future-directed and twice continuously differentiable; $\dot{\gamma}$ denotes its tangent vector field giving the normalised time direction and $a=\ddot{\gamma}$ is the acceleration. Now let $v$ be any differentiable vector field along $\gamma$. Then $v$ is Fermi-Walker transported along $\gamma$ (cf. for example [24]) if and only if

$$
\dot{v}=(\dot{\gamma} \cdot v) a-(a \cdot v) \dot{\gamma}
$$

If $v(0) \cdot \dot{\gamma}(0)=0$ at the initial point $t=0$, then it follows from (2.1) that $v \cdot \dot{\gamma}=0$ all along $\gamma$; for $\frac{d}{d t}(v \cdot \dot{\gamma})=\dot{v} \cdot \dot{\gamma}+v \cdot \ddot{\gamma}=-(a \cdot v)+(v \cdot a)=0$ in view of the normalisation $\dot{\gamma}^{2}=1$. Similarly one can conclude the following from (2.1): Two differentiable vector fields $v$ and $w$ that are FermiWalker transported along $\gamma$ are mutually metric-orthonormal (i.e. $v^{2}=-1, w^{2}=-1, v \cdot w=0$ ) along all of $\gamma$ if and only if they have that property at some single point of $\gamma$. This implies that, if at the initial point $t=0$ a set of pairwise metric-orthonormal vectors $\kappa_{j}(0), j=1,2,3$, is chosen such that $\left(\dot{\gamma}(0), \kappa_{1}(0), \kappa_{2}(0), \kappa_{3}(0)\right)$ forms a four-dimensional Lorentz frame (tetrad) affixed at $\gamma(0)$, and if $\kappa_{j}(t)$ denotes the vectors obtained from Fermi-Walker transporting the $\kappa_{j}(0)$ along $\gamma$ from $\gamma(0)$ to $\gamma(t), j=1,2,3$, then $\left(\dot{\gamma}(t), \kappa_{1}(t), \kappa_{2}(t), \kappa_{3}(t)\right)$ is again a four-dimensional Lorentz frame, affixed at $\gamma(t)$. As a matter of fact, the frame obtained by Fermi-Walker transport along $\gamma$ is differentiable. Moreover, it is not difficult to see that there is a continuous function of proper orthochronous Lorentz

transformations $t \mapsto \Lambda(t) \in \mathscr{L}_{+}^{\uparrow}$ inducing the Fermi-Walker transport along $\gamma$, i.e. it transforms the frame $\left(\dot{\gamma}(0), \kappa_{1}(0), \kappa_{2}(0), \kappa_{3}(0)\right)$ onto the frame $\left(\dot{\gamma}(t), \kappa_{1}(t), \kappa_{2}(t), \kappa_{3}(t)\right), t \geq 0$. 
Next, we consider the motion of the laboratory, which is initially at rest in the spherical region $\boldsymbol{L}_{0}=\left\{x: x_{0}=0,|\boldsymbol{x}-\boldsymbol{o}|<r\right\}$. As explained, we want to describe its motion by the Fermi-Walker transport of $\boldsymbol{L}_{0}$ along the world line $\gamma$. Thus, at proper time $t>0$ of the observer, we are led to assign to the laboratory the region

$$
\boldsymbol{L}_{t}=\left\{\gamma(t)+\sum_{j=1}^{3} y_{j} \kappa_{j}(t):|\boldsymbol{y}|<r\right\}=\gamma(t)+\Lambda(t)\left(\boldsymbol{L}_{0}-\gamma(0)\right)
$$

where $\boldsymbol{y}=\left(y_{1}, y_{2}, y_{3}\right)$ are the spatial coordinates relative to the position of the observer in his current Lorentz system. This corresponds to the conceived experimental situation, where the laboratory is being rigidly dragged along the worldline $\gamma$, keeping its spherical shape relative to the observer.

If the laboratory undergoes an accelerated motion, i.e. if $a=\ddot{\gamma} \neq 0$, the various points in the laboratory follow worldlines with acceleration other than $a$ and so there are tidal forces acting on the laboratory. Apart from the restrictions on the acceleration of $\gamma$ stemming from preserving rigidity of a realistic laboratory against the tidal forces, there is an a priory restriction on the acceleration that derives from the requirement that any point in the laboratory must follow a timelike, futuredirected worldline. In other words, the laboratory is to be represented by a congruence of timelike, future-directed worldlines. To see what this restriction amounts to, let us parametrise the worldlines of points of the laboratory according to

$$
\gamma_{\boldsymbol{y}}(t) \doteq \gamma(t)+\sum_{j=1}^{3} y_{j} \kappa_{j}(t), \quad|\boldsymbol{y}|<r .
$$

Since the $\kappa_{j}$ are Fermi-Walker transported along $\gamma=\gamma_{\boldsymbol{o}}$, Eqn. (2.1) implies

$$
\dot{\gamma} \boldsymbol{y}(t)=\dot{\gamma}(t)+\sum_{j=1}^{3} y_{j} \dot{\kappa}_{j}(t)=\left(1-\sum_{j=1}^{3} y_{j} a(t) \cdot \kappa_{j}(t)\right) \dot{\gamma}(t),
$$

hence all the $\gamma_{\boldsymbol{y}}$ are timelike and future-directed if and only if $\sum_{j=1}^{3} y_{j} a(t) \cdot \kappa_{j}(t)<1$ for all $|\boldsymbol{y}|<r$ at all times $t \geq 0$. In view of $\dot{\gamma} \cdot a=0$ and as the $\kappa_{j}(t), j=1,2,3$, form an orthonormal basis of the hyperplane that lies metric-orthogonal to $\dot{\gamma}(t)$, this implies that the acceleration must satisfy the condition $r \sqrt{-a^{2}}<1 \widehat{=} c^{2}$ all along any admissible worldline $\gamma$.

It may be worth inserting some numbers into this condition to see that realistic laboratories are quite far from that bound. According to references compiled in [35], among the highest accelerations known so far are $1.9 \cdot 10^{9} \mathrm{~m} / \mathrm{s}^{2}$ for protons in the LHC, $7 \cdot 10^{12} \mathrm{~m} / \mathrm{s}^{2}$ at the surface of a neutron star, and $8.8 \cdot 10^{13} \mathrm{~m} / \mathrm{s}^{2}$ for protons at the Fermilab accelerator. As $c^{2}=9 \cdot 10^{16} \mathrm{~m}^{2} / \mathrm{s}^{2}$, a perfectly rigid laboratory could still extend about $r=10^{3} \mathrm{~m}$ without violating the above bound even at these extreme accelerations. Material stresses would of course deform or destroy a realistic laboratory at much lower acceleration scales. The present limit for instrumentation acceleration is at about $1.6 \cdot 10^{5} \mathrm{~m} / \mathrm{s}^{2}$. 
So the conclusion of this discussion is the insight that it is meaningful (i) to consider spatially extended rigid laboratories undergoing acceleration with time scale fixed by the clock of the observer and (ii) to describe the motion of the laboratory by the action of Poincaré transformations, which are parametrised by the proper time of the observer, cf. Eqn. (2.2).

\section{Macroscopic stability of the vacuum against acceleration}

Having clarified the description of moving laboratories, we will show now that the macroscopic properties of the vacuum state found by an observer do not change under the influence of arbitrary accelerations along his world line $\gamma$. The observables in the laboratory region $\boldsymbol{L}_{t}$ at his proper time $t \geq 0$ are described by elements of the algebra $\mathcal{A}\left(\mathcal{O}_{t}\right)$, where the double cone $\mathcal{O}_{t}$ is the causal completion of the spatial region $\boldsymbol{L}_{t}$. Note that the indexing of the algebras by double cones is merely a matter of notational convenience. It is justified by the fact that the underlying Weyl operators are defined on the quotient $\mathcal{D}\left(\mathcal{O}_{t}\right) / \square \mathcal{D}\left(\mathbb{R}^{4}\right)$, which corresponds to the space of Cauchy data of the wave equation with support in $\boldsymbol{L}_{t}$.

According to Eqn. (2.2) one has $\boldsymbol{L}_{t}=\Gamma(t) \boldsymbol{L}_{0}$, where $\Gamma(t) \doteq(\gamma(t)-\Lambda(t) \gamma(0), \Lambda(t), 1) \in \mathscr{W}_{+}^{\uparrow}$. This entails the corresponding relations for the causal completions, $\mathcal{O}_{t}=\Gamma(t) \mathcal{O}_{0}, t \geq 0$. It then follows from the covariant action of the group $\mathscr{W}_{+}^{\uparrow}$ on the algebras that $\mathcal{A}\left(\mathcal{O}_{t}\right)=\mathcal{A}\left(\Gamma(t) \mathcal{O}_{0}\right)=\alpha_{\Gamma(t)}\left(\mathcal{A}\left(\mathcal{O}_{0}\right)\right)$, $t \geq 0$. Thus for all admissible world lines $\gamma$ satisfying the constraint on the acceleration given in the preceding section, the motion of the observables in the laboratory can be described by the automorphic action of the group. Being interested in persistent macroscopic properties, the observer will be led to analyse the states with his observables $\alpha_{\Gamma(t)}(A)$ at asymptotic times $t \rightarrow \infty$. As a matter of fact, these observables form central sequences whose limits can thus be interpreted as classical observables. For the proof we need the following two lemmas, the first one being of geometric nature.

Lemma 3.1. Let $t \mapsto \gamma(t)$ be any admissible world line of an observer and let $\mathcal{O}_{t}, t \geq 0$, be the causal completion of his Fermi-Walker transported laboratory region of radius $r$, where $r \sqrt{-a^{2}} \leq v^{2}<1$ all along $\gamma$. There exist an open double cone $\mathcal{O} \subset \mathbb{R}^{4}$ and a lightlike vector $l$ such that for any given bounded region $\mathcal{B} \subset(\mathcal{O}+\mathbb{R} l) \doteq \bigcup_{u \in \mathbb{R}}(\mathcal{O}+u l) \subset \mathbb{R}^{4}$ the region $\mathcal{O}_{t}$ is timelike separated from $\mathcal{B}$ for sufficiently large $t>0$.

Proof. The lower tip of the double cone $\mathcal{O}_{t}$ moves along the world line $t \mapsto \eta(t) \doteq(\gamma(t)-r \dot{\gamma}(t))$, hence $\mathcal{O}_{t} \subset\left(V_{+}+\eta(t)\right)$, where $V_{+}$denotes the forward lightcone, $t \geq 0$. It therefore suffices to establish the existence of $\mathcal{O}$ and $\mathbb{R} l$, as described in the statement, by replacing $\mathcal{O}_{t}$ with $\eta(t), t \geq 0$. Shifting the origin in $\mathbb{R}^{4}$, one may also assume that $\eta(0)=0$. Since $\dot{\eta}(t)=(\dot{\gamma}(t)-r a(t))$ and $\dot{\eta}(t)^{2}=\left(1+r^{2} a(t)^{2}\right) \geq\left(1-v^{2}\right)>0$ it follows that $\eta(t) \in V_{+}$and $\eta(t)^{2} \geq\left(1-v^{2}\right) t^{2}, t \geq 0$. Depending 
on the characteristics of the world line $\eta$ one must distinguish two cases by means of the characteristic hyperplanes $H_{s}(\boldsymbol{s})=\left\{x: x_{0}-\boldsymbol{s} \boldsymbol{x}=s\right\}$ for arbitrary unit vectors $\boldsymbol{s} \in S^{2}$ and times $s \geq 0$.

The first type of world line $t \mapsto \eta(t)$ has the property that it crosses all of these hyperplanes in the course of time. Let $t_{s}(s)$ be the time where $\eta\left(t_{s}(s)\right) \in H_{s}(s)$. Note that $t_{s}(s)$ is unique and that for $t>t_{s}(s)$ one has $\eta(t) \in F_{s}(s) \doteq\left\{x: x_{0}-\boldsymbol{s} \boldsymbol{x}>s\right\}$, the future of $H_{s}(\boldsymbol{s})$, since the worldline is timelike and future directed and the hyperplanes do not contain any timelike directions. Moreover, for fixed $s \geq 0$, the map $s \mapsto t_{s}(s)$ is continuous since the world line $\eta$ is continuous. Hence there exists the supremum $t_{s} \doteq \sup _{\boldsymbol{s} \in S^{2}} t_{s}(\boldsymbol{s})$ since $S^{2}$ is compact. Thus for $t>t_{s}$ one has $\eta(t) \in \bigcap_{\boldsymbol{s} \in S^{2}} F_{s}(\boldsymbol{s})=\left(V_{+}+(s, \mathbf{0})\right)$. Since $s \geq 0$ was arbitrary and since for any given bounded region $\mathcal{B} \subset \mathbb{R}^{4}$ one has $\mathcal{B} \subset\left(-V_{+}+(s, \mathbf{0})\right)$ for sufficiently large $s \geq 0$ it follows that $\eta(t)$ and therefore also $\mathcal{O}_{t}$ is timelike separated from $\mathcal{B}$ for $t>t_{s}$.

The second type of world line $t \mapsto \eta(t)$ has the property that there is some hyperplane $H_{s_{0}}\left(\boldsymbol{s}_{0}\right)$ which it does not cross. Hence $\eta(t) \in V_{+} \bigcap P_{s_{0}}\left(\boldsymbol{s}_{0}\right), t \geq 0$, where $P_{s_{0}}\left(\boldsymbol{s}_{0}\right) \doteq\left\{x: x_{0}-\boldsymbol{s}_{0} \boldsymbol{x}<s_{0}\right\}$ is the past of $H_{s_{0}}\left(\boldsymbol{s}_{0}\right)$. One then obtains for the lightlike vector $l \doteq\left(1, \boldsymbol{s}_{0}\right)$ and $u \in \mathbb{R}$

$$
(\eta(t)-u l)^{2}=\eta(t)^{2}-2 u\left(\eta_{0}(t)-\boldsymbol{s}_{0} \boldsymbol{\eta}(t)\right) \geq\left(1-v^{2}\right) t^{2}-2|u| s_{0}, \quad t \geq 0 .
$$

It follows from this estimate that for any double cone $\mathcal{O} \subset\left(-V_{+}\right)$and $u$ varying in any bounded interval $\mathbb{I} \subset \mathbb{R}$, the regions $(\mathcal{O}+u l), u \in \mathbb{I}$, are timelike separated from $\eta(t)$ for sufficiently large $t \geq 0$. This completes the proof of the statement.

In the second lemma we show that the algebra of observables associated with the lightlike cylinder $(\mathcal{O}+\mathbb{R} l)$, defined in the preceding statement, is irreducibly represented in the GNS representation $\left(\pi_{0}, \mathcal{H}_{0}, \Omega_{0}\right)$ induced by the inertial vacuum state $\omega_{0}$. Here $\mathcal{H}_{0}$ denotes the familiar Fock space, $\Omega_{0} \in \mathcal{H}_{0}$ the Fock vacuum and $\pi_{0}$ the homomorphism mapping the elements of the algebra $\mathcal{A}\left(\mathbb{R}^{4}\right)$ to bounded operators in $\mathcal{B}\left(\mathcal{H}_{0}\right)$. One then has the equality of expectation values $\omega_{0}(A)=\left\langle\Omega_{0}, \pi_{0}(A) \Omega_{0}\right\rangle$, $A \in \mathcal{A}\left(\mathbb{R}^{4}\right)$. We also recall that there is a continuous unitary representation $U_{0}$ of the extended Poinaré group on $\mathcal{H}_{0}$ satisfying $U_{0}(\Upsilon) \pi_{0}(A) U_{0}(\Upsilon)^{-1}=\pi_{0}\left(\alpha_{\Upsilon}(A)\right), A \in \mathcal{A}(\mathcal{O})$ and $U_{0}(\Upsilon) \Omega_{0}=\Omega_{0}, \Upsilon \in \mathscr{W}_{+}^{\uparrow}$. Moreover, the joint spectrum of the generators of the spacetime translations $U_{0} \uparrow \mathbb{R}^{4}$ is contained in the closed forward lightcone. The following statement, whose proof is given for completeness, is a well-known consequence of these properties.

Lemma 3.2. Let $\mathcal{O} \subset \mathbb{R}^{4}, l \in \mathbb{R}^{4}$, be any double cone and lightlike translation. respectively, and let $\mathcal{A}(\mathcal{O}+\mathbb{R} l)$ be the algebra generated by $\mathcal{A}(\mathcal{O}+u l), u \in \mathbb{R}$. This algebra is irreducibly represented in the vacuum representation $\left(\pi_{0}, \mathcal{H}_{0}, \Omega_{0}\right)$, viz. the commutant of $\pi_{0}(\mathcal{A}(\mathcal{O}+\mathbb{R} l))$ in $\mathcal{B}(\mathcal{H})$ consists of multiples of the identity, $\pi_{0}(\mathcal{A}(\mathcal{O}+\mathbb{R} l))^{\prime}=\mathbb{C} 1$. 
Proof. Let $U_{0}(u l), u \in \mathbb{R}$, be the unitaries implementing the subgroup of lightlike translations $\mathbb{R} l$. Due to covariance, $U_{0}(u l) \pi_{0}(\mathcal{A}(\mathcal{O}+\mathbb{R} l)) U_{0}(u l)^{-1}=\pi_{0}\left(\alpha_{u l}(\mathcal{A}(\mathcal{O}+\mathbb{R} l))\right)=\pi_{0}(\mathcal{A}(\mathcal{O}+\mathbb{R} l)), u \in \mathbb{R}$, and, by the Reeh-Schlieder property of the vacuum [14, Sec. II.5.3], $\pi_{0}(\mathcal{A}(\mathcal{O}+\mathbb{R} l)) \Omega_{0} \subset \mathcal{H}_{0}$ is a dense subspace of $\mathcal{H}_{0}$. Now let $Z \in \pi_{0}(\mathcal{A}(\mathcal{O}+\mathbb{R} l))^{\prime}$, then for any $A \in \mathcal{A}(\mathcal{O}+\mathbb{R} l)$ and $u \in \mathbb{R}$

$$
\left\langle\Omega_{0}, Z U_{0}(u l) \pi_{0}(A) \Omega_{0}\right\rangle=\left\langle\Omega_{0}, Z \pi_{0}\left(\alpha_{u l}(A)\right) \Omega_{0}\right\rangle=\left\langle\Omega_{0}, \pi_{0}\left(\alpha_{u l}(A)\right) Z \Omega_{0}\right\rangle=\left\langle\Omega_{0}, \pi_{0}(A) U_{0}(u l)^{-1} Z \Omega_{0}\right\rangle,
$$

as a consequence of covariance and the invariance of $\Omega_{0}$ under the action of $U_{0}(u l), u \in \mathbb{R}$. The unitary group of lightlike translations $u \mapsto U_{0}(u l), u \in \mathbb{R}$, has a positive generator and the ray of $\Omega_{0}$ is the unique invariant subspace of $\mathcal{H}_{0}$ under its action. (The latter fact is a well-known consequence of the representation theory of the Poincaré group in four spacetime dimensions.) It therefore follows from the preceding equality by Fourier analysis with regard to $u \in \mathbb{R}$ that the matrix elements do not dependent on $u \in \mathbb{R}$, hence $\left\langle\Omega_{0}, \pi_{0}(A) Z \Omega_{0}\right\rangle=\left\langle\Omega_{0}, \pi_{0}(A) \Omega_{0}\right\rangle\left\langle\Omega_{0}, Z \Omega_{0}\right\rangle, A \in \mathcal{A}(\mathcal{O}+\mathbb{R} l)$. The Reeh-Schlieder property of $\Omega_{0}$ then implies $Z=\left\langle\Omega_{0}, Z \Omega_{0}\right\rangle 1$, completing the proof.

We are now in the position to prove the main result of this section.

Proposition 3.3. Let $t \mapsto \gamma(t)$ be any admissible world line as in Lemma[3.1 and let $t \mapsto \Gamma(t) \in \mathscr{W}_{+}^{\uparrow}$ be the family of Poincaré transformations inducing the Fermi-Walker transport of the laboratory region, $\mathcal{O}_{t}=\Gamma(t) \mathcal{O}_{0}, t \geq 0$. There exist in the vacuum representation $\left(\pi_{0}, \mathcal{H}_{0}, \Omega_{0}\right)$ of $\mathcal{A}\left(\mathbb{R}^{4}\right)$ the limits

$$
\lim _{t \rightarrow \infty} \pi_{0}\left(\alpha_{\Gamma(t)}(A)\right)=\omega_{0}(A) 1, \quad A \in \mathcal{A}\left(\mathcal{O}_{0}\right)
$$

in the weak operator topology.

Proof. Given any $A \in \mathcal{A}\left(\mathcal{O}_{0}\right)$ one has $\alpha_{\Gamma(t)}(A) \in \mathcal{A}(\Gamma(t) \mathcal{O})=\mathcal{A}\left(\mathcal{O}_{t}\right), t \geq 0$. Now according to Lemma 3.1 there exist a double cone $\mathcal{O}$ and a lightlike vector $l \in \mathbb{R}^{4}$ such that for any finite interval $\mathbb{I} \subset \mathbb{R}$ the regions $\mathcal{O}_{t}$ and $(\mathcal{O}+\mathbb{I} l)$ are timelike separated for sufficiently large $t \geq 0$. It therefore follows from Huygens' principle that the operators $\alpha_{\Gamma(t)}(A)$ commute with any given operator $B \in \mathcal{A}(\mathcal{O}+\mathbb{R} l)$ in the limit $t \rightarrow \infty$.

Proceeding to the vacuum representation one makes use of the fact that for any given $A \in \mathcal{A}\left(\mathcal{O}_{0}\right)$ the family of operators $\pi_{0}\left(\alpha_{\Gamma(t)}(A)\right), t \geq 0$, is uniformly bounded and thus has weak limit points in $\mathcal{B}\left(\mathcal{H}_{0}\right)$. Let $t_{n} \geq 0, n \in \mathbb{N}$, be any sequence such that there exists the limit $Z \doteq \lim _{n \rightarrow \infty} \pi_{0}\left(\alpha_{\Gamma\left(t_{n}\right)}(A)\right)$ in the weak operator topology. As a consequence of Huygens' principle one has $\left[Z, \pi_{0}(B)\right]=0$ for any $B \in \mathcal{A}(\mathcal{O}+\mathbb{R} l)$ and since $\pi_{0}(\mathcal{A}(\mathcal{O}+\mathbb{R} l))$ is irreducible, cf. Lemma [3.2, it follows that $Z=z 1$ for some $z \in \mathbb{C}$. Now

$$
z=\left\langle\Omega_{0}, Z \Omega_{0}\right\rangle=\lim _{t \rightarrow \infty}\left\langle\Omega_{0}, \pi_{0}\left(\alpha_{\Gamma\left(t_{n}\right)}(A)\right) \Omega_{0}\right\rangle=\lim _{t \rightarrow \infty} \omega_{0}\left(\alpha_{\Gamma\left(t_{n}\right)}(A)\right)=\omega_{0}(A),
$$


where the last equality obtains from the invariance of the vacuum under the action of the Poincaré group. Thus all weak limit points of $\pi_{0}\left(\alpha_{\Gamma(t)}(A)\right)$ for $t \rightarrow \infty$ coincide with $\omega_{0}(A) 1$, so this family of operators is convergent in the weak operator topology for $A \in \mathcal{A}\left(\mathcal{O}_{0}\right)$, as stated.

This result shows that quantum effects, leading to excitations of the vacuum described by vectors in $\mathcal{H}_{0}$, are suppressed in the limit of large times: the observables $\alpha_{\Gamma(t)}(A), t \geq 0$, form central sequences in the vacuum representation which converge weakly and have sharp (non-fluctuating) limits due to the asymptotic abelianess of the dynamics. As a matter of fact, by taking suitable time averages one can also accomplish their convergence in the strong operator topology. The limits of these sequences give information about persistent macroscopic properties of the underlying states. In view of the fact that they do not depend on the world line $\gamma$, we conclude that an accelerated observer will not register any macroscopic effects of the acceleration in the vacuum state. In particular, he does not experience the vacuum state as a thermal gas, respectively bath, contrary to statements made in the literature, cf. [12, p. 167], [29, p. 3721], [32, p. 115]. For such a gas would leave a trace in the asymptotic expectation values of some observable $A$ which differs from its vacuum expectation value $\omega_{0}(A)$.

We conclude this section by indicating how these central sequences of observables may be used in order to determine the temperature of states, thereby complementing the discussion in [4]. Within the present model, the inertial equilibrium states in the chosen Lorentz system are described by functionals $\omega_{T}, T>0$, on $\mathcal{A}\left(\mathbb{R}^{4}\right)$. They are fixed by (linear extension from) the expectation values

$$
\omega_{T}(W(f)) \doteq e^{-v_{T}(f, f) / 2}, \quad f \in \mathcal{D}_{\square}\left(\mathbb{R}^{4}\right)
$$

where $v_{T}(f, f) \doteq(2 \pi)^{-3} \int d^{4} p \varepsilon\left(p_{0}\right) \delta\left(p^{2}\right)\left(1-e^{-p_{0} / T}\right)^{-1} \widehat{f}(-p) \widehat{f}(p)$ is the thermal two-point function of the free field. Proceeding to the corresponding GNS-representations $\left(\pi_{T}, \mathcal{H}_{T}, \Omega_{T}\right)$ one can show that for inertial motion $t \mapsto \gamma(t) \doteq(t, \mathbf{0})$ one has $\lim _{t \rightarrow \infty} \pi_{T}\left(\alpha_{\gamma(t)}(A)\right)=\omega_{T}(A) 1, A \in \mathbb{R}^{4}$, in the weak operator topology. (This is a well known consequence of the fact that the KMS states $\omega_{T}$ are faithful and have the mixing property, i.e. they describe pure phases.) For fixed $A$ the mapping $T \mapsto \omega_{T}(A)$ describes the equation of state of the intensive quantity measured by $A$ as a function of the inertial temperature. Thus the observer may calibrate his observables in the inertial equilibrium states and use them as empirical thermometers also when he is in motion. According to the preceding results he would then come to the conclusion that in the presence of acceleration the temperature of the vacuum remains to be zero, in accordance with the results in [4]. 


\section{Macroscopic properties of accelerated equilibrium states}

If an observer undergoes some constant acceleration of modulus a he will be able to prepare in his laboratory equilibrium states and study their macroscopic properties. In this section we determine the predictions which the present model makes about his findings. It will turn out that, in contrast to the inertial situation, the global parameter $T_{\mathrm{a}}$ characterising the accelerated equilibrium states cannot be interpreted as temperature anymore. In fact, disregarding the inertial vacuum, the temperature varies spatially within these states.

In order to keep the notation simple, we assume that the observer moves with constant acceleration a $>0$ along the world line $t \mapsto \gamma_{\mathrm{a}}(t)=(\operatorname{sh}(\mathrm{ta}) / \mathrm{a}, \operatorname{ch}(\mathrm{ta}) / \mathrm{a}, 0,0), t \geq 0$. As is well-known and can also be inferred from the discussion in Sec. 2, the maximally possible initial laboratory region is, in this special case, the half space $\boldsymbol{L}_{0}=\left\{x: x_{0}=0, x_{1}>0\right\}$. At later times this region is Fermi-Walker transported to $\boldsymbol{L}_{t}=\left\{x: x_{0}=t h(t \mathrm{a}) x_{1}>0\right\}=\Lambda_{\mathrm{a}}(t) \boldsymbol{L}_{0}$, where $\Lambda_{\mathrm{a}}(t) \in \mathscr{L}_{+}^{\uparrow}$ are the boosts fixed by the given worldline, $t \geq 0$. The causal completion of each of these laboratory regions is the wedge shaped region $\mathcal{W} \doteq\left\{x: x_{1}>\left|x_{0}\right|\right\} \subset \mathcal{M}$ which is stable under the action of the boosts. Let us mention as an aside that one should not think of this wedge region as Rindler space, having an insurmountable boundary at its edge (the apparent horizon). For that idea might induce one to take the Rindler vacuum as a (global) reference state, which is disjoint from the inertial vacuum and leads to an interpretation of the theory in terms of "Rindler quanta". Such an interpretation would not correspond to the situation treated here, where a Minkowski space based observer enters a spacecraft in order to perform experiments with his local observables.

The equilibrium states which, in principle, can be prepared by the accelerated observer in his laboratory are stationary and passive [17] and hence are also described by KMS states $\omega_{T_{\mathrm{a}}}, T_{\mathrm{a}}>0$, on the algebra $\mathcal{A}(\mathcal{W})$ with regard to the automorphic action $\alpha_{\Lambda_{\mathrm{a}}(t)}, t \in \mathbb{R}$, of the time translations. Note that the index a will be used throughout in order to distinguish quantities related to the latter dynamics. The KMS states are fixed by (linear extension from) the expectation values

$$
\omega_{T_{\mathrm{a}}}(W(f)) \doteq e^{-v_{T_{\mathrm{a}}}(f, f) / 2}, \quad f \in \mathcal{D}_{\square}(\mathcal{W})
$$

where the thermal two-point function $v_{T_{a}}$ is now given by

$$
v_{T_{\mathrm{a}}}(f, f) \doteq(2 \pi)^{-4} \int d u\left(1-e^{-u / T_{\mathrm{a}}}\right)^{-1} \int d v e^{-i u v} \int d^{4} p \varepsilon\left(p_{0}\right) \delta\left(p^{2}\right) \widehat{f}(-p) \widehat{f}\left(\Lambda_{\mathrm{a}}(v) p\right) .
$$

Since we are not aware of a reference where it has been shown that the functionals $\omega_{T_{\mathrm{a}}}, T_{\mathrm{a}}>0$, are KMS states (satisfying the condition of positivity) in case of the free massless scalar field we provide a proof in an appendix. The well-known fact that for the special value $T_{\mathrm{a}}=\mathrm{a} / 2 \pi$ the corresponding 
state $\omega_{T_{\mathrm{a}}}$ coincides with the restriction of the inertial vacuum to the wedge algebra, $\omega_{T_{\mathrm{a}}}=\omega_{0} \uparrow \mathcal{A}(\mathcal{W})$, is commonly interpreted as formal evidence for the Unruh effect [11, 21, 30].

In analogy to the results established in the preceding section one can show that observables that are localised in relatively compact regions of $\mathcal{W}$ form central sequences at asymptotic times, which have sharp limit values in the GNS-representations induced by the KMS states $\omega_{T_{a}}$ on $\mathcal{A}(\mathcal{W}), T_{a}>0$. The proof is based on standard arguments and given here for completeness.

Proposition 4.1. Let $\omega_{T_{\mathrm{a}}}, T_{\mathrm{a}}>0$, be any KMS state, defined above relative to the automorphic action of the dynamics $\alpha_{\Lambda_{\mathrm{a}}(t)}, t \in \mathbb{R}$, on the algebra $\mathcal{A}(\mathcal{W})$, and let $\left(\pi_{T_{\mathrm{a}}}, \mathcal{H}_{T_{\mathrm{a}}}, \Omega_{T_{\mathrm{a}}}\right)$ be the corresponding GNS-representation. There exist the limits

$$
\lim _{t \rightarrow \infty} \pi_{T_{\mathrm{a}}}\left(\alpha_{\Lambda_{\mathrm{a}}(t)}(A)\right)=\omega_{T_{\mathrm{a}}}(A) 1, \quad A \in \mathcal{A}(\mathcal{W}),
$$

in the weak operator topology.

Proof. The crucial step in the argument is the proof that the state $\omega_{T_{\mathrm{a}}}$ is mixing, i.e. for any pair of operators $A, B \in \mathcal{A}(\mathcal{W})$ one has $\lim _{t \rightarrow \infty} \omega_{T_{\mathrm{a}}}\left(B \alpha_{\Lambda_{\mathrm{a}}(t)}(A)\right)=\omega_{T_{\mathrm{a}}}(B) \omega_{T_{\mathrm{a}}}(A)$. This property implies that $\lim _{t \rightarrow \infty} \pi_{T_{\mathrm{a}}}\left(\alpha_{\Lambda_{\mathrm{a}}(t)}(A)\right) \Omega_{T_{\mathrm{a}}}=\omega_{T_{\mathrm{a}}}(A) \Omega_{T_{\mathrm{a}}}, A \in \mathcal{A}(\mathcal{W})$, in the sense of weak convergence in $\mathcal{H}_{T_{\mathrm{a}}}$. Since the family of operators $\pi_{T_{\mathrm{a}}}\left(\alpha_{\Lambda_{\mathrm{a}}(t)}(A)\right), t \geq 0$, is uniformly bounded, hence has weak limit points, and KMS states are separating for the weak closure of the represented algebra, the statement then follows. For the proof of the mixing property one makes use of the fact that the elements of $\mathcal{A}(\mathcal{W})$ are linear combinations of Weyl operators, so it suffices to consider the functions $t \mapsto \omega_{T_{\mathrm{a}}}\left(W(g) \alpha_{\Lambda_{\mathrm{a}}(t)}(W(f))\right)$, $f, g \in \mathcal{D}_{\square}(\mathcal{W})$. Applying the Weyl relations one gets

$$
\omega_{T_{\mathrm{a}}}\left(W(g) \alpha_{\Lambda_{\mathrm{a}}(t)}(W(f))\right)=e^{-\kappa\left(g, f_{\Lambda_{\mathrm{a}}(t)}-1\right) / 2} \omega_{T_{\mathrm{a}}}\left(W\left(g+f_{\Lambda_{\mathrm{a}}(t)^{-1}}\right)\right)=e^{-v_{T_{\mathrm{a}}}\left(g, f_{\Lambda_{\mathrm{a}}(t)}-1\right)} e^{-v_{T_{\mathrm{a}}}(g, g) / 2} e^{-v_{T_{\mathrm{a}}}(f, f) / 2} .
$$

Furthermore, making use of the group law $\Lambda_{\mathrm{a}}(v) \Lambda_{\mathrm{a}}(t)=\Lambda_{\mathrm{a}}(v+t)$, one obtains

$$
v_{T_{\mathrm{a}}}\left(g, f_{\Lambda_{\mathrm{a}}(t)^{-1}}\right)=(2 \pi)^{-4} \int d u e^{i t u}\left(1-e^{-u / T_{\mathrm{a}}}\right)^{-1} \int d v e^{-i u v} \int d^{4} p \varepsilon\left(p_{0}\right) \delta\left(p^{2}\right) \widehat{g}(-p) \widehat{f}\left(\Lambda_{\mathrm{a}}(v) p\right) .
$$

It is shown in the appendix that $u \mapsto \int d v e^{-i u v} \int d^{4} p \varepsilon\left(p_{0}\right) \delta\left(p^{2}\right) \widehat{g}(-p) \widehat{f}\left(\Lambda_{\mathrm{a}}(v) p\right)$ is a test function that vanishes at $u=0$ for any choice of $f, g \in \mathcal{D}_{\square}(\mathcal{W})$. The Riemann-Lebesgue lemma then implies $\lim _{t \rightarrow \infty} v_{T_{\mathrm{a}}}\left(g, f_{\Lambda_{\mathrm{a}}^{-1}(t)}\right)=0$ and hence $\lim _{t \rightarrow \infty} \omega_{T_{\mathrm{a}}}\left(W(g) \alpha_{\Lambda_{\mathrm{a}}(t)}(W(f))\right)=\omega_{T_{\mathrm{a}}}(W(g)) \omega_{T_{\mathrm{a}}}(W(f))$, completing the proof.

Thus, in the presence of constant acceleration, an observer can still describe the macroscopic properties of the equilibrium states in his laboratory in terms of relations $T_{\mathrm{a}} \mapsto \omega_{T_{\mathrm{a}}}(A)$ involving his intensive observables, which are parametrised by the equilibrium parameter $T_{\mathrm{a}}>0$. In contrast to the inertial situation, the equilibrium states are not spatially homogeneous, however. Note that the semigroup of spatial translations $\mathbb{R}_{+}^{3} \doteq\left\{\boldsymbol{y}: y_{1} \geq 0\right\}$ maps the wedge $\mathcal{W}$ into itself. 
Proposition 4.2. Let $\omega_{T_{\mathrm{a}}}, T_{\mathrm{a}}>0$, be any KMS state, defined above relative to the automorphic action of the dynamics $\alpha_{\Lambda_{\mathrm{a}}(t)}, t \in \mathbb{R}$ on the algebra $\mathcal{A}(\mathcal{W})$. If $\omega_{T_{\mathrm{a}}}$ is invariant under the semigroup of spatial translations, viz. $\omega_{T_{\mathrm{a}}} \circ \alpha_{\boldsymbol{y}}=\omega_{T_{\mathrm{a}}}, \boldsymbol{y} \in \mathbb{R}_{+}^{3}$, then $T_{\mathrm{a}}=\mathrm{a} / 2 \pi$ and $\omega_{T_{\mathrm{a}}}$ coincides with the restriction of the inertial vacuum state $\omega_{0}$ to $\mathcal{A}(\mathcal{W})$.

Proof. The boosts $\mathcal{B}_{\mathrm{a}} \doteq\left\{\Lambda_{\mathrm{a}}(t): t \in \mathbb{R}\right\}$ and spatial translations $\mathbb{R}_{+}^{3}=\left\{\boldsymbol{y}: y_{1} \geq 0\right\}$ generate the semigroup $\overline{\mathcal{W}} \rtimes \mathcal{B}_{\mathrm{a}}$, where $\overline{\mathcal{W}}=\left\{y: y_{1} \geq\left|y_{0}\right| \geq 0\right\} \subset \mathbb{R}^{4}$ denotes the cone of spacetime translations acting as endomorphisms on $\mathcal{W}$. Since $\omega_{T_{a}}$ is invariant under the automorphic actions of the boosts and spatial translations, it is also invariant under the action of the semigroup, i.e. $\omega_{T_{\mathrm{a}}} \circ \alpha_{\Sigma}=\omega_{T_{\mathrm{a}}}, \Sigma \in \overline{\mathcal{W}} \rtimes \mathcal{B}_{\mathrm{a}}$. Proceeding to the GNS-representation $\left(\pi_{T_{\mathrm{a}}}, \mathcal{H}_{T_{\mathrm{a}}}, \Omega_{T_{\mathrm{a}}}\right)$ induced by $\omega_{T_{\mathrm{a}}}$, this implies that there exists a continuous unitary representation $U_{T_{\mathrm{a}}}: \overline{\mathcal{W}} \rtimes \mathcal{B}_{\mathrm{a}} \rightarrow \mathcal{B}\left(\mathcal{H}_{T_{\mathrm{a}}}\right)$ given by $U_{T_{\mathrm{a}}}(\Sigma) \pi_{T_{\mathrm{a}}}(A) \Omega_{T_{\mathrm{a}}} \doteq \pi_{T_{\mathrm{a}}}\left(\alpha_{\Sigma}(A)\right) \Omega_{T_{\mathrm{a}}}, A \in \mathcal{A}(\mathcal{W})$. (The isometries $U_{T_{\mathrm{a}}}(\Sigma)$ have a dense range due to the Reeh-Schlieder property of $\Omega_{T_{\mathrm{a}}}$, cf. [25].) Since the group $(\overline{\mathcal{W}}-\overline{\mathcal{W}})=\mathbb{R}^{4}$ is abelian and the boosts $\mathcal{B}_{\text {a }}$ normalise $\overline{\mathcal{W}}$, one can consistently extend the unitary representation $U_{\text {a }}$ of $\overline{\mathcal{W}} \rtimes \mathcal{B}_{\text {a }}$ to $\mathbb{R}^{4} \rtimes \mathcal{B}_{\mathrm{a}}$, putting $U_{\mathrm{a}}\left(x-y, \Lambda_{\mathrm{a}}\right) \doteq U_{\mathrm{a}}(x) U_{\mathrm{a}}(y)^{-1} U_{\mathrm{a}}\left(\Lambda_{\mathrm{a}}\right)$ for $x, y \in \overline{\mathcal{W}}, \Lambda_{\mathrm{a}} \in \mathcal{B}_{\mathrm{a}}$.

Consider now the lightlike translations $l_{ \pm}=( \pm l, l, 0,0), l \in \mathbb{R}$, satisfying $\Lambda_{\mathrm{a}}(t) l_{ \pm}=e^{ \pm t a} l_{ \pm}, t \in \mathbb{R}$. Thus $\alpha_{l_{ \pm}}(\mathcal{A}(\mathcal{W}))=\mathcal{A}\left(\mathcal{W}+l_{ \pm}\right) \subset \mathcal{A}(\mathcal{W})$ for any given $l>0$ and $\alpha_{\Lambda_{\mathrm{a}}(t)}\left(\mathcal{A}\left(\mathcal{W}+l_{ \pm}\right)\right) \subset \mathcal{A}\left(\mathcal{W}+l_{ \pm}\right)$for $\pm t \geq 0$ and $l>0$. Since $\omega_{T_{\mathrm{a}}}$ is a KMS state, $t \rightarrow U_{\mathrm{a}}\left(\Lambda_{\mathrm{a}}(t)\right)$ is (after rescaling of $t$ ) the modular group associated with the pair $\left(\pi_{T_{\mathrm{a}}}(\mathcal{A}(\mathcal{W}))^{-}, \Omega_{T_{\mathrm{a}}}\right)$, where the bar ${ }^{-}$denotes closure in the weak operator topology. Hence for any $l>0$ the algebras $U_{\mathrm{a}}\left(l_{ \pm}\right) \pi_{T_{\mathrm{a}}}(\mathcal{A}(\mathcal{W}))^{-} U_{\mathrm{a}}\left(l_{ \pm}\right)^{-1} \subset \pi_{T_{\mathrm{a}}}(\mathcal{A}(\mathcal{W}))^{-}$form "halfsided modular inclusions" which implies that the one-parameter groups $l \mapsto U_{\mathrm{a}}\left(l_{ \pm}\right), l \in \mathbb{R}$ have positive and negative generators, respectively, cf. [33]. Thus, putting $l=1 / 2$, the generator of the group of inertial time translations $t \mapsto U_{\mathrm{a}}\left(t\left(l_{+}-l_{-}\right)\right), t \in \mathbb{R}$, is positive and $\Omega_{T_{\mathrm{a}}} \in \mathcal{H}_{T_{\mathrm{a}}}$ is a ground state for it. Since the free massless scalar field has a unique scale invariant inertial ground state, viz. the inertial vacuum $\omega_{0}$, this shows that the given state $\omega_{T_{a}}$ coincides with the restriction $\omega_{0} \uparrow \mathcal{A}(\mathcal{W})$, proving that $T_{\mathrm{a}}=\mathrm{a} / 2 \pi$.

The preceding result can be established in the present model also by explicit computations. Our general argument, however, shows that the spatial inhomogeneity of equilibrium states in a uniformly accelerated laboratory is a model independent feature. In the case at hand, we are dealing with equilibrium states of a relativistic ideal gas, where one expects a one-to-one correspondence between the local (empirical) temperature and the asymptotic expectation values of suitable intensive observables $A$. The fact that $\boldsymbol{x} \mapsto \omega_{T_{\mathrm{a}}}\left(\alpha_{\boldsymbol{x}}(A)\right)$ is in general not constant therefore already suggests that the global parameter $T_{\mathrm{a}}$ cannot be interpreted as temperature. This point will be substantiated in the subsequent discussion, where it is shown that in the present model all equilibrium states coincide at 
sufficiently large distance from the edge of the wedge $\mathcal{W}$ (the horizon). For the proof of this assertion we make use of the following facts.

(i) All KMS states $\omega_{T_{\mathrm{a}}}, T_{\mathrm{a}}>0$, on $\mathcal{A}(\mathcal{W})$ are invariant under the automorphic action of the dilations $(0,1, \lambda) \in \mathscr{W}_{+}^{\uparrow}, \lambda>0$, i.e. $\omega_{T_{\mathrm{a}}} \circ \alpha_{\lambda}=\omega_{T_{\mathrm{a}}}$ in short hand notation. This is easily inferred from the definition of the states and the fact that the dilations commute with Lorentz transformations.

(ii) The restrictions of the states $\omega_{T_{\mathrm{a}}} \uparrow \mathcal{A}(\mathcal{O}), T_{\mathrm{a}}>0$, to the algebra of any relatively compact region $\mathcal{O} \subset \mathcal{W}$ are normal with respect to each other, i.e. they are continuous on the unit ball of the algebra in the weak operator topology induced by any one of these states. This follows from the fact that the KMS states $\omega_{T_{\mathrm{a}}}, T_{\mathrm{a}}>0$, are quasifree Hadamard states [19] and that such states are locally normal with respect to each other [31]. The latter fact implies that the states can locally be interpreted in terms of ensembles described by density matrices in the Fock space of the inertial vacuum. But, similarly to the inertial case, different KMS states are not normal (in fact, disjoint) with respect to each other on the whole algebra $\mathcal{A}(\mathcal{W})$.

(iii) Finally, let $\mathcal{O}_{R, r} \subset \mathcal{W}$ be the double cone centred at $\boldsymbol{o}_{R}=(0, R, 0,0)$ with spherical basis of radius $0<r<R$ at time $x_{0}=0$. The dilations act on this region according to $\lambda \mathcal{O}_{R, r}=\mathcal{O}_{\lambda R, \lambda r}$, $\lambda>0$. Because of the invariance of the thermal states under the automorphic action of the dilations one obtains for their local norm distance $\left\|\omega_{T_{\mathrm{a}}}-\omega_{T_{\mathrm{a}}^{\prime}}\right\|_{\mathcal{O}_{R, r}} \doteq \sup _{A \in \mathcal{A}\left(\mathcal{O}_{R, r}\right)}\left|\omega_{T_{\mathrm{a}}}(A)-\omega_{T_{\mathrm{a}}^{\prime}}(A)\right| /\|A\|$ the equalities $\left\|\omega_{T_{\mathrm{a}}}-\omega_{T_{\mathrm{a}}^{\prime}}\right\|_{\mathcal{O}_{R, r}}=\left\|\omega_{T_{\mathrm{a}}} \circ \alpha_{\lambda}-\omega_{T_{\mathrm{a}}^{\prime}} \circ \alpha_{\lambda}\right\|_{\mathcal{O}_{R, r}}=\left\|\omega_{T_{\mathrm{a}}}-\omega_{T_{\mathrm{a}}^{\prime}}\right\|_{\mathcal{O}_{\lambda R, \lambda r}}, \lambda>0$. After these preparations we can establish the following fact, which relies on arguments given by Roberts in [18].

Proposition 4.3. Let $\omega_{T_{\mathrm{a}}}, \omega_{T_{\mathrm{a}}^{\prime}}$ be any pair of $K M S$ states on the algebra $\mathcal{A}(\mathcal{W})$ with regard to the automorphic action of the dynamics $\alpha_{\Lambda_{\mathrm{a}}(t)}, t \in \mathbb{R}$. Then $\lim _{r / R \rightarrow 0}\left\|\omega_{T_{\mathrm{a}}}-\omega_{T_{\mathrm{a}}^{\prime}}\right\|_{\mathcal{O}_{R, r}}=0$. In particular, the norm distance vanishes in this limit for any $T_{\mathrm{a}}>0$ and fixed $T_{\mathrm{a}}^{\prime}=a / 2 \pi$, where $\omega_{T_{\mathrm{a}}^{\prime}}$ coincides with the restriction of the inertial vacuum $\omega_{0}$ to the wedge algebra, $\omega_{T_{a}^{\prime}}=\omega_{0} \uparrow \mathcal{A}(\mathcal{W})$.

Proof. Because of the triangle inequality for the norm distance it suffices to prove the statement for the special case $\omega_{T_{\mathrm{a}}^{\prime}}=\omega_{0} \uparrow \mathcal{A}(\mathcal{W})$. Moreover, inserting into the equality $\left\|\omega_{T_{\mathrm{a}}}-\omega_{0}\right\|_{\mathcal{O}_{R, r}}=\left\|\omega_{T_{\mathrm{a}}}-\omega_{0}\right\|_{\mathcal{O}_{\lambda R, \lambda r}}$ the special value $\lambda=1 / R$, one only needs to estimate the norm distances $\left\|\omega_{T_{\mathrm{a}}}-\omega_{0}\right\|_{\mathcal{O}_{1, r / R}}$. Copying the argument in [18, this is accomplished by making use of the fact that the intersection of the double cones $\mathcal{O}_{1, r / R}, r / R>0$, consists of the single point $(0,1,0,0) \in \mathcal{W}$. Hence the intersection of the corresponding weakly closed algebras in the GNS representations $\left(\pi_{0} . \mathcal{H}_{0}, \Omega_{0}\right)$ induced by $\omega_{0}$ consists of multiples of the identity, $\bigcap_{r / R>0} \pi_{0}\left(\mathcal{A}\left(\mathcal{O}_{1, r / R}\right)\right)^{-}=\mathbb{C} 1$. The latter relation says that there exist no non-trivial bounded operators that are localised at a point, a classical result due to Wightman [34]. Now there exists for any $r / R>0$ an operator $A_{r / R} \in \mathcal{A}\left(\mathcal{O}_{1, r / R}\right)$ with norm $\left\|A_{r / R}\right\|=1$ such that $\left\|\omega_{T_{\mathrm{a}}}-\omega_{0}\right\|_{\mathcal{O}_{1, r / R}} \leq\left(\left|\omega_{T_{\mathrm{a}}}\left(A_{r / R}\right)-\omega_{0}\left(A_{r / R}\right)\right|+(r / R)\right)$. Since all weak limit points of the uniformly 
bounded sequence of operators $\pi_{0}\left(A_{r / R}\right), r / R \rightarrow 0$, are contained in $\bigcap_{r / R>0} \pi_{0}\left(\mathcal{A}\left(\mathcal{O}_{1, r / R}\right)\right)^{-}$and hence are multiples of the identity, and since the states $\omega_{T_{\mathrm{a}}}$ are locally normal with respect to $\omega_{0}$, this implies $\lim _{r / R \rightarrow 0}\left\|\omega_{T_{\mathrm{a}}}-\omega_{0}\right\|_{\mathcal{O}_{1, r / R}}=0$ and the statement follows.

This result shows that the states $\omega_{T_{\mathrm{a}}}, T_{\mathrm{a}}>0$, can practically not be discriminated from the inertial vacuum $\omega_{0}$ by observations in regions of arbitrarily large radius $r$ that are separated from the edge of the wedge $\mathcal{W}$ (the apparent horizon) by a distance $R \gg r$. Thus in spite of the fact that these states correspond to different equilibrium parameters $T_{\mathrm{a}}$, one clearly must assign to them in these remote regions the same temperature as to the inertial vacuum. On the other hand, the state $\omega_{\mathrm{a} / 2 \pi}=\omega_{0}\lceil\mathcal{A}(\mathcal{W})$ is spatially homogeneous with regard to all local observables in the laboratory and hence has the same temperature everywhere. Since the Tolman-Ehrenfest law [27, 10] implies that the local temperature in a uniformly accelerated equilibrium state is proportional to its inverse distance from the horizon, the temperature must be zero everywhere in state $\omega_{a / 2 \pi}$. This fact substantiates our assertion that the temperature of the inertial vacuum remains to be zero in uniformly accelerated systems and that the global equilibrium parameters $T_{\mathrm{a}}$, even if corrected by redshift factors, cannot directly be interpreted as temperature of accelerated equilibrium states. We refer the reader to [4] for a definition of observables indicating the effective local temperature of equilibrium states in the present setting, cf. also [5] and the subsequent concluding remarks.

\section{Conclusions}

In the present article we have studied the macroscopic effects of acceleration on equilibrium states, as seen by an observer in a rigid, spatially extended laboratory. The macroscopic properties of these states are determined by local observables in the respective laboratory system, which form central sequences at asymptotic times. These sequences have sharp limits, hence quantum fluctuations are suppressed. It turned out that acceleration does not affect the macroscopic properties of an inertial vacuum state. Irrespective of the accelerated, possibly erratic motion of the laboratory, the observer will find the same macroscopic properties of the vacuum as an inertial observer. In particular, he will not find himself immersed in a thermal gas, respectively heat bath.

We have also shown that the equilibrium parameter $T_{\mathrm{a}}$, distinguishing the KMS states in a uniformly accelerated laboratory, cannot offhandedly be interpreted as temperature. Disregarding the particular value $T_{\mathrm{a}}=\mathrm{a} / 2 \pi$, the states are inhomogeneous and coincide at sufficiently large distances from the horizon of the observer with the inertial vacuum. Hence, in spite of the fact that these states correspond to different equilibrium parameters $T_{\mathrm{a}}$, one must assign to them the same temperature in these remote regions. Moreover, as a consequence of the Tolman-Ehrenfest law, the parameter 
$T_{\mathrm{a}}=\mathrm{a} / 2 \pi$ attributed to the spatially homogeneous vacuum may not be regarded as its temperature either; in fact, the temperature must vanish throughout this state according to this law.

An operationally meaningful definition of temperature, based on the concept of local thermometer observables, was proposed in [4. Proceeding to the idealisation of pointlike observables, the simplest example of a local thermometer is, in the present setting, the normal ordered square of the underlying free field, $\Theta(x) \doteq 12: \phi^{2}:(x)$. The numerical factor is determined by calibration in the inertial equilibrium states $\omega_{T}$, yielding the expectation values $\omega_{T}(\Theta(x))=T^{2}, T \geq 0$. Taking suitably regularised time limits of this thermometer observable in the representation induced by any given accelerated KMS state $\omega_{T_{\mathrm{a}}}, T_{\mathrm{a}}>0$, one obtains for the local temperature $T_{\mathrm{a}}\left(x_{1}\right)$ at distance $x_{1}$ from the horizon the result [4]

$$
T_{\mathrm{a}}^{2}\left(x_{1}\right)=\omega_{T_{\mathrm{a}}}\left(\Theta\left(x_{1}\right)\right)=\left(\mathrm{a} x_{1}\right)^{-2}\left(T_{\mathrm{a}}^{2}-(\mathrm{a} / 2 \pi)^{2}\right) .
$$

Thus, in accordance with the Tolman-Ehrenfest law, one finds that a $x_{1} T_{\mathrm{a}}\left(x_{1}\right)=$ const, $x_{1}>0$, in the given states. Note that the constant appearing in this law does not coincide with the KMS parameter $T_{\mathrm{a}}$, contrary to the common ad hoc definition of local temperatures, where the global KMS parameters $T_{\mathrm{a}}$ are divided through the local redshift factors a $x_{1}$. In fact, the constant is modified by a contribution due to the Unruh temperature, which vanishes only in the classical limit. So in this approach the temperature attributed to the inertial vacuum in the accelerated laboratory turns also out to be zero, $T_{\mathrm{a} / 2 \pi}\left(x_{1}\right)=0$ for $x_{1}>0$. This result is in accordance with the present findings, where we did not rely on an a priori concept of local thermometers.

Some contingent objection against these observations derives from the fact that microscopic probes, which are locally coupled to accelerated KMS states in order to model "local thermometers", are driven to Gibbs ensembles corresponding to one and the same parameter $T_{\mathrm{a}}$ which characterises the respective underlying macroscopic KMS state. This fact is often taken as an argument that $T_{\mathrm{a}}$ ought to be interpreted as temperature of that state. Yet, as has been outlined in the introduction, any local coupling does not only induce the transfer of thermal energy (heat) between the KMS states and the probe; it inevitably creates also excitations of these states because of the quantum nature of the coupling [20, p 334] (KMS states are faithful, cf. [14]). These quantum induced excitations gain energy by the acceleration that is partly transferred to the probes. The probes therefore indicate temperatures $T_{\mathrm{a}}$ which are higher than the local (redshifted) temperature of the underlying KMS state, $T_{\mathrm{a}}>$ a $x_{1} T_{\mathrm{a}}\left(x_{1}\right)$, cf. relation (5.1).

This mechanism is also effective in the inertial vacuum state. There the value $T_{\mathrm{a}}=\mathrm{a} / 2 \pi$ indicated by the probe is entirely due to the energetic contributions of quantum induced excitations, there are no contributions coming from a "vacuum gas". Phrased differently, instead of indicating the temperature of the vacuum, the probe indicates its own temperature at asymptotic times, which is raised by local 
quantum effects during the measuring process [20]. This conclusion is confirmed by the observation that the value of $T_{\mathrm{a}}$ does not depend on the position of the probe within the laboratory, i.e. on its particular world line. The latter fact can be extracted from remarks in [7, pp 6531-6532] about the arbitrary choice of form factors, determining the position of the probe within the laboratory. As already noticed by these authors, this feature is at variance with the conventional interpretation of the Unruh effect. So the Unruh effect is a quantum induced systematic contribution, which appears in certain specific measuring procedures of temperature, but, as explained, may be avoided by others [4]. Its popular thermal interpretation is not tenable, however.

The upshot of the present investigation, going beyond the case considered here, is the insight that the parameters $T_{*}$, characterising KMS states, may in general not directly be interpreted as temperatures and that probes (Unruh-de Witt detectors), which reliably determine these parameters, may therefore not be regarded as perfect thermometers. In the presence of acceleration or, equivalently, gravitation and also of curvature, cf. [3, 23, the parameters $T_{*}$ subsume information about the local temperature of the underlying equilibrium states as well as of these other local data, cf. relation (5.1). Phrased differently, the value of $T_{*}$ dictates the relation between these local parameters which is required to obtain global equilibrium. For inertial systems or small accelerations and curvatures, $T_{*}$ may safely be identified with temperature; to give an example, for terrestrial acceleration a $=9.8 \mathrm{~m} / \mathrm{s}^{2}$ and local temperature $T=300{ }^{\circ} \mathrm{K}$ the corresponding KMS parameter $T_{\mathrm{a}}$ indicated by a probe would be, according to theory, about $2.710^{-42}{ }^{0} \mathrm{~K}$ higher. But in the neighbourhood of huge masses or black holes these systematic effects can no longer be neglected and the interpretation of thermal properties of states should then no longer be based directly on the KMS parameters $T_{*}$. Further investigations of this issue therefore seem warranted.

\section{Appendix}

We supply in this appendix the proof that the functionals $\omega_{T_{\mathrm{a}}}$ on the algebra $\mathcal{A}(\mathcal{W})$, generated by the free scalar massless field, which are defined in relations (4.1) and (4.2), are KMS states with regard to the automorphic action of the dynamics $\alpha_{\Lambda_{\mathrm{a}}(t)}, t \in \mathbb{R}$. In doing so we rely on the well-known fact that the state $\omega_{\mathrm{a} / 2 \pi}$ coincides with the restriction of the inertial vacuum state $\omega_{0}\lceil\mathcal{A}(\mathcal{W})$, which satisfies

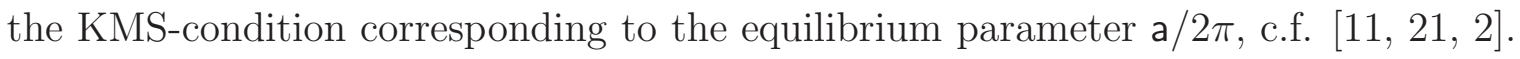

We begin by noting that the commutator function of the free massless theory can be presented in the form $\kappa(f, g)=\langle f, g\rangle_{0}-\langle g, f\rangle_{0}$, where $\langle f, g\rangle_{0}$ denotes the scalar product of the single particle vectors $|f\rangle_{0},|g\rangle_{0} \in \mathcal{H}_{0}, f, g \in \mathcal{D}_{\square}\left(\mathbb{R}^{4}\right)$; these are generated from $\Omega_{0}$ by the smeared free field operators in the GNS representation $\left(\pi_{0}, \mathcal{H}_{0}, \Omega_{0}\right)$ induced by $\omega_{0}$. Restricting these quantities to test functions $f, g \in \mathcal{D}_{\square}(\mathcal{W})$ we make use of the following facts. 
(i) For any given $f, g \in \mathcal{D}_{\square}(\mathcal{W})$, having relatively compact supports in $\mathcal{W}$, the support of $g_{\Lambda_{\mathrm{a}}(t)^{-1}}$ is timelike separated from the support of $f$ for sufficiently large $|t|$, cf. the proof of Lemma 3.1. Thus, because of Huygens' principle, the function $t \mapsto \kappa\left(f, g_{\Lambda_{\mathrm{a}}(t)^{-1}}\right), t \in \mathbb{R}$, has compact support. It is also arbitrarily often differentiable (since the free field is an operator-valued distribution) and hence is a test function.

(ii) The boosts are unitarily implemented in the vacuum representation, viz. $U_{0}\left(\Lambda_{\mathrm{a}}(t)\right)=e^{i t a K}, t \in \mathbb{R}$, where $K$ is the canonical generator of the boosts. Since $\Omega_{0}$ is invariant under the action of these unitary operators, one obtains $\left\langle f, g_{\Lambda_{\mathrm{a}}(t)^{-1}}\right\rangle_{0}=\left\langle f, e^{i t a K} g\right\rangle_{0}, t \in \mathbb{R}$. Moreover, as $\omega_{0}\lceil\mathcal{A}(\mathcal{W})$ satisfies the KMS-condition with respect to the action of $\alpha_{\Lambda_{\mathrm{a}}(t)}$ for the equilibrium parameter a/2 $\pi$, it follows that the vectors $|f\rangle_{0},|g\rangle_{0}$ are in the domain of $e^{-\pi K}$ and that the KMS boundary condition can be presented for the single particle vectors in the form $\left\langle e^{-\pi K} f, e^{-\pi K} g_{\Lambda_{\mathrm{a}}(t)^{-1}}\right\rangle_{0}=\left\langle g_{\Lambda_{\mathrm{a}}(t)^{-1}}, f\right\rangle_{0}, t \in \mathbb{R}$. So one arrives at the equality $\left(\left\langle f, e^{i t a K} g\right\rangle_{0}-\left\langle e^{-\pi K} f, e^{i t a K} e^{-\pi K} g\right\rangle_{0}\right)=\kappa\left(f, g_{\Lambda_{\mathrm{a}}(t)^{-1}}\right), t \in \mathbb{R}$, for any $f, g \in \mathcal{D}_{\square}(\mathcal{W})$.

(iii) One now makes use of the spectral decomposition of the selfadjoint generator $K$, which has Lebesgue absolutely continuous spectrum on the single particle space. Denoting by $E(k), k \in \mathbb{R}$, its spectral resolution, one obtains the equality $\int e^{i t a k}\left(1-e^{-2 \pi k}\right) d\langle f, E(k) g\rangle_{0}=\kappa\left(f, g_{\Lambda_{\mathrm{a}}(t)^{-1}}\right), t \in \mathbb{R}$. Since $t \mapsto \kappa\left(f, g_{\Lambda_{\mathrm{a}}(t)^{-1}}\right)$ is a test function, its Fourier transform $k \mapsto \sqrt{2 \pi}\left(1-e^{-2 \pi k / \mathrm{a}}\right) d\langle f, E(k / \mathrm{a}) g\rangle_{0} / d k$ is a (Schwartz) test function as well and vanishes at the origin for any $f, g \in \mathcal{D}_{\square}(\mathcal{W})$. Incidentally, this establishes the property used in the last part of the proof of Proposition 4.1.

(iv) Now the KMS boundary condition given above implies $e^{-2 \pi k} d\langle f, E(k) g\rangle_{0}=d\langle g, E(-k) f\rangle_{0}$ on $\mathbb{R}$ for any $f, g \in \mathcal{D}_{\square}(\mathcal{W})$. Since the function $k \mapsto\left(1-e^{-2 \pi k}\right)\left(1-e^{-\mathrm{a} k / T_{\mathrm{a}}}\right)^{-1}$ is positive and continuous for any $T_{\mathrm{a}}>0$, it follows that the real bilinear forms, given in (4.2), can be presented as

$$
v_{T_{\mathrm{a}}}(f, f)=\int\left(1-e^{-2 \pi k}\right)\left(1-e^{-\mathrm{a} k / T_{\mathrm{a}}}\right)^{-1} d\langle f, E(k) f\rangle_{0}, \quad f \in \mathcal{D}_{\square}(\mathcal{W}),
$$

and hence are well-defined. Moreover, they can canonically be extended in both entries to sesquilinear forms on the complex linear space $\left(\mathcal{D}_{\square}(\mathcal{W})+i \mathcal{D}_{\square}(\mathcal{W})\right)$. Since the functions $k \mapsto\langle f, E(k) f\rangle_{0}$ are continuous and monotonously increasing for any $f \in\left(\mathcal{D}_{\square}(\mathcal{W})+i \mathcal{D}_{\square}(\mathcal{W})\right)$, they determine positive measures on $\mathbb{R}$. Hence $v_{T_{\mathrm{a}}}$ defines a positive scalar product on $\left(\mathcal{D}_{\square}(\mathcal{W})+i \mathcal{D}_{\square}(\mathcal{W})\right)$ for any $T_{\mathrm{a}}>0$. By the KMS boundary condition and a change of variables one also obtains for any $f, g \in \mathcal{D}_{\square}(\mathcal{W})$

$$
\begin{aligned}
v_{T_{\mathrm{a}}}(f, g)-v_{T_{\mathrm{a}}}(g, f) & =\int\left(1-e^{-2 \pi k}\right)\left(1-e^{-\mathrm{a} k / T_{\mathrm{a}}}\right)^{-1}\left(d\langle f, E(k) g\rangle_{0}-d\langle g, E(k) f\rangle_{0}\right) \\
& =\int\left(\left(1-e^{-2 \pi k}\right)\left(1-e^{-\mathrm{a} k / T_{\mathrm{a}}}\right)^{-1}-\left(1-e^{2 \pi k}\right)\left(1-e^{\mathrm{a} k / T_{\mathrm{a}}}\right)^{-1} e^{-2 \pi k}\right) d\langle f, E(k) g\rangle_{0} \\
& =\int\left(1-e^{-2 \pi k}\right) d\langle f, E(k) g\rangle_{0}=\kappa(f, g) .
\end{aligned}
$$


Hence $v_{T_{\mathrm{a}}}$ defines a two-point function of the free massless scalar field for any $T_{\mathrm{a}}>0$.

(v) It remains to prove that the functions $t \mapsto v_{T_{\mathrm{a}}}\left(f, g_{\Lambda_{\mathrm{a}}(t)^{-1}}\right)$ comply with the KMS-condition. Making use of step (iv), one obtains for $f, g \in \mathcal{D}_{\square}(\mathcal{W})$ and $t \in \mathbb{R}$

$$
v_{T_{\mathrm{a}}}\left(f, g_{\Lambda_{\mathrm{a}}(t)^{-1}}\right)=\int e^{i t \mathrm{a} k}\left(1-e^{-a k / T_{\mathrm{a}}}\right)^{-1}\left(1-e^{-2 \pi k}\right) d\langle f, E(k) g\rangle_{0} .
$$

Bearing in mind that $k \mapsto\left(1-e^{-2 \pi k}\right) d\langle f, E(k) g\rangle_{0} / d k$ is a test function that vanishes at $k=0$, it is then apparent that $t \mapsto v_{T_{\mathrm{a}}}\left(f, g_{\Lambda_{\mathrm{a}}(t)^{-1}}\right)$ can be continued to a function that is continuous and bounded on the strip $\left\{z: 0 \leq \operatorname{Im} z \leq 1 / T_{\mathrm{a}}\right\}$ and analytic in its interior. Its boundary value at the upper rim of this strip is given by

$$
\begin{aligned}
& \int e^{i t \mathrm{a} k} e^{-a k / T_{\mathrm{a}}}\left(1-e^{-a k / T_{\mathrm{a}}}\right)^{-1}\left(1-e^{-2 \pi k}\right) d\langle f, E(k) g\rangle_{0} \\
= & \int e^{-i \mathrm{ta} k} e^{a k / T_{\mathrm{a}}}\left(1-e^{a k / T_{\mathrm{a}}}\right)^{-1}\left(1-e^{2 \pi k}\right) d\langle f, E(-k) g\rangle_{0} \\
= & \int e^{-i t \mathrm{a} k}\left(1-e^{-a k / T_{\mathrm{a}}}\right)^{-1}\left(1-e^{-2 \pi k}\right) e^{-2 \pi k} d\langle g, E(k) f\rangle_{0}=v_{T_{\mathrm{a}}}\left(g_{\Lambda_{\mathrm{a}}(t)^{-1}}, f\right),
\end{aligned}
$$

proving the KMS property of $v_{T_{\mathrm{a}}}, T_{\mathrm{a}}>0$.

(vi) After these preparations it follows by standard arguments, cf. for example [16], that the functionals $\omega_{T_{\mathrm{a}}}$, defined in equation (4.1) in terms of the two-point function $v_{T_{\mathrm{a}}}$, are states on the algebra $\mathcal{A}(\mathcal{W})$ of the free massless scalar field. For, making use of the Weyl relations, one has for any $f_{i}, f_{k} \in \mathcal{D}_{\square}(\mathcal{W})$, $i, k=1, \ldots, N$,

$$
\omega_{T_{\mathrm{a}}}\left(W\left(f_{i}\right)^{*} W\left(f_{k}\right)\right)=e^{\kappa\left(f_{i}, f_{k}\right) / 2} e^{-v_{T_{a}}\left(f_{k}-f_{i}, f_{k}-f_{i}\right) / 2}=e^{-v_{T_{a}}\left(f_{i}, f_{i}\right) / 2} e^{v_{T_{a}}\left(f_{i}, f_{k}\right)} e^{-v_{T_{a}}\left(f_{k}, f_{k}\right) / 2} .
$$

Since the Hadamard products $v_{T_{a}}\left(f_{i}, f_{k}\right)^{n}$ of the positive matrix $v_{T_{a}}\left(f_{i}, f_{k}\right), i, k=1, \ldots, N$, are again positive matrices for any $n \in \mathbb{N}$, it is apparent that the functional $\omega_{T_{a}}$ satisfies the condition of positivity. Moreover, the exponential function is entire analytic, hence the functions

$$
t \mapsto \omega_{T_{\mathrm{a}}}\left(W(f) \alpha_{\Lambda_{\mathrm{a}}(t)}(W(g))=e^{-v_{T_{a}}(f, f) / 2} e^{-v_{T_{a}}\left(f, g_{\left.\Lambda_{\mathrm{a}}(t)-1\right)}\right.} e^{-v_{T_{a}}(g, g) / 2}, \quad f, g \in \mathcal{D}_{\square}(\mathcal{W})\right.
$$

satisfy the KMS-condition for the dynamics $\alpha_{\Lambda_{\mathrm{a}}(t)}, t \in \mathbb{R}$, since the two-point function $v_{T_{a}}$ does. This completes the proof that $\omega_{T_{\mathrm{a}}}$ is a KMS state for any temperature $T_{\mathrm{a}}>0$.

We conclude this appendix by noting that, by similar arguments, one can also establish the existence of a ground state for this dynamics in case of the free massless scalar field. 


\section{Acknowledgment}

We acknowledge correspondence with Stephen A. Fulling, William G. Unruh and Robert M. Wald which induced us to include in this article remarks on the relation between our results and the TolmanEhrenfest law. We are also grateful to Ko Sanders for constructive comments on a preliminary version.

\section{References}

[1] Bell, J.S., Hughes, R.J., Leinaas, J.M., "The Unruh effect in extended thermometers" Z. Phys. C28 (1985) 75-80

[2] Bisognano, J.J, Wichmann, E.H., "On the duality theory for a Hermitean scalar field", J. Math. Phys. 16 (1975) 985-1007

[3] Buchholz, D., Schlemmer, J., "Local temperature in curved spacetime", Class. Quant. Grav. 24 (2007) F25F31

[4] Buchholz, D., Solveen, Ch., "Unruh effect and the concept of temperature", Class. Quant. Grav. 30 (2013) 085011

[5] Buchholz, D., Verch, R., "Unruh versus Tolman: On the heat of acceleration", e-print arXiv:1505.01686

[6] Crispino, L.C.B., Higuchi, A., Matsas, G.E.A., "The Unruh effect and its applications", Reviews of Modern Physics 80 (2008) 787-838

[7] De Bièvre, M., Merkli, M., "The Unruh effect revisited", Class. Quant. Grav. 23 (2006) 6525-6541

[8] Dereziński, J., Jakšić, V., "Return to equilibrium for Pauli-Fierz systems", Ann. Henri Poincaré 4 (2003) 739-793

[9] Earman, J., "The Unruh effect for philosophers", Stud. Hist. Philos. Mod. Phys. 42 (2011) 81-97

[10] Ehrenfest, P., Tolman, R.C., "Temperature equilibrium in a static gravitational field". Phys. Rev. 36 (1930) 1791-1798

[11] Fulling, S.A., "Nonuniqueness of canonical field quantization in Riemannian space-time", Phys. Rev. D7 (1973) 2850-2862

[12] Fulling, S.A., Ruijsenaars, S.N.M., "Temperature, periodicity and horizons", Phys. Reports 152 (1987) 135176.

[13] Ford, G.W., O'Connell, R.F., "Is there Unruh radiation?", Phys. Lett. A 350 (2006) 1726 
[14] Haag, R., Local Quantum Physics, 2nd Ed., Springer-Verlag, Berlin, Heidelberg, New York, 1996

[15] Hepp, K., "Quantum theory of measurement and macroscopic observables", Helv. Phys. Acta 45 (1972) 237248

[16] Manuceau, J., Verbeure, A., "Quasi-free states of the CCR-Algebra and Bogoliubov transformations". Commun. Math. Phys. 4 (1968) 293-302

[17] Pusz, W., Woronowicz, S.L., "Passive states and KMS states for general quantum systems", Commun. Math. Phys. 58 (1978) 273-290

[18] Roberts, J.E., "Some applications of dilation invariance to structural questions in the theory of local observables", Commun. Math. Phys. 37 (1974) 273-286

[19] Sahlmann, H., Verch, R., "Passivity and microlocal spectrum condition", Commun. Math. Phys. 214 (2000) 705-731

[20] Sciama, D.W., Candelas, P., Deutsch, D., "Quantum field theory, horizons and thermodynamics", Advances in Physics 30 (1981) 327-366

[21] Sewell, G., "Quantum fields on manifolds: PCT and gravitationally induced thermal states", Annals Phys. 141 (1982) 201-224

[22] Sewell, G., Quantum Mechanics and Its Emergent Macrophysics, Princeton University Press, 2002

[23] Solveen, Ch., "Local thermal equilibrium and KMS states in curved spacetime", Class. Quant. Grav. 29 (2012) 245015

[24] Straumann, N., General Relativity, 2nd Ed., Springer Verlag, Dordrecht, Berlin, Heidelberg, New York, (2012)

[25] Strohmaier, A., Verch, R., Wollenberg, M., "Microlocal analysis of quantum fields on curved spacetimes: Analytic wavefront sets and Reeh-Schlieder theorems", J. Math. Phys. 43 (2002) 5514-5530

[26] Takagi, S., "Vacuum noise and stress induced by uniform acceleration Hawking-Unruh effect in Rindler manifold of arbitrary dimension" Prog. Theor. Phys. Supplement 88 (1986) 1-142

[27] Tolman, R.C., "On the weight of heat and thermal equilibrium in general relativity", Phys. Rev. 35 (1930) 904-924

[28] Unruh, W.G., "Notes on black-hole evaporation", Phys. Rev. D14 (1976) 870-892

[29] Unruh, W.G., "Thermal bath and decoherence of Rindler spacetime”, Phys. Rev, D 46 (1992) 3271-3277 
[30] Unruh, W.G., Wald, R.M., "What happens when an accelerating observer detects a Rindler particle", Phys. Rev. D 29 (1984) 1047-1056

[31] Verch, R., "Local definiteness, primarity and quasiequivalence of quasifree Hadamard quantum states in curved spacetime", Commun. Math. Phys. 160 (1994) 507-536

[32] Wald, R.M., "Quantum field theory in curved spacetime and black hole thermodynamics", Chicago Lectures in Physics, University of Chicago Press, 1994

[33] Wiesbrock, H.-W., "Half-sided modular inclusions of von-Neumann-algebras", Comm. Math. Phys. 157 (1993) 83-92 Erratum-ibid. 184 (1997) 683-685

[34] Wightman, A.S., "La théorie quantique locale et la thorie quantique des champs", Ann. Inst. Henri Poincare 1 (1964) 403-420

[35] http://en.wikipedia.org/wiki/Orders_of_magnitude_(acceleration) 